\title{
Vinculación del constructivismo con el enfoque socio-psicológico para el estudio de los usuarios de unidades de información: un primer acercamiento teórico
}

\author{
Tania Peña Vera *
}

Artículo recibido:

11 de mayo de 2004.

Artículo aceptado:

21 de enero de 2005.

\section{RESUMEN}

Mediante una investigación documental se analizan los principales postulados del constructivismo y del enfoque socio-psicológico para estudiar los usuarios de unidades y los servicios de información. Al hallar entre ellos puntos coincidentes se concluyó que el primero de estos conceptos constituye parte del basamento teórico sobre el cual se sustenta el segundo, en el sentido de que ambos le otorgan especial importancia al contexto del individuo y a sus conocimientos previos, como antecedente necesario para iniciar cualquier acción formativa o informativa. Tal integración de perspectivas responde a la complejidad creciente de los saberes, que tienden a desdibujar las fronteras de los conocimientos que otrora se manejaban parceladamente.

Escuela de Bibliotecología y Archivología de la Universidad del Zulia, Venezuela. tanialirena@yahoo.com. 
Palabras Clave: Constructivismo; Enfoque sociopsicológico; Usuarios; Servicios de información; Unidades de información.

\begin{abstract}
Concatenation of Constructivism with the Socio-Psycological Approach to the study of information unit users: a first theoretical approach

Tania Peña-Vera

The main postulates of Constructivism and the SocioPsychological approach are analyzed through documentary research in order to study the users of information units. Since some agreement was found, it was concluded that Constructivism is part of the theoretical basis on which the Socio-Psychological Approach stands, for both give special importance to context of the individual and to his previous knowledge as the necessary premise before starting any formative or informative action. Such integration of perspectives responds to the growing complexity of our cognition, which tends to diminish knowledge frontiers which formerly were handled separately.
\end{abstract}

Keywords: Constructivism; Socio-Psychological approach; Users; Information services; Information units.

\title{
INTRODUCCIÓN
}

L a constante evolución de los conceptos y sus significados, y los cambios en los estilos de vida que acarrea el moldeamiento de los comportamientos, suscitan una serie de interrogantes acerca del tipo de formación que demandan las actuales y futuras generaciones; en ese sentido, la educación representa un instrumento poderoso para operar cambios sociales que se reflejen en el pensamiento del colectivo, orientados a asumir la complejidad creciente, lo inesperado y acelerado de las transformaciones, derribar las barreras de los enfoques tradicionalistas que dispersan los saberes, acortan la visión y fragmentan el conocimiento. 
Morin $^{1}$ añade que las determinaciones sociales, económicas y políticas apuntan a encerrar el conocimiento en un marasmo de normas, prohibiciones, rigideces y bloqueos, que impiden que lo inesperado se adentre en nuestros preceptos, teorías y estamentos, los cuales hemos heredado y nos brindan estabilidad en la medida en que permanecen inmóviles. Ante tal situación es preciso que la educación sirva como impulsora de las grandes interrogantes sobre el mundo, el hombre y el conocimiento, y promueva la búsqueda de respuestas mediante la reflexión, la ecologización y la integración de puntos de vista que generen teorías nuevas, abiertas, críticas, racionales y permeables a las reformas.

Explica este autor que la tendencia es pues a religar conocimientos anteriores con los actuales; a integrar las teorías de diferentes sectores científicos que de otro modo permanecerían divorciadas; a lograr el equilibrio entre la unidad y la diversidad humana, y fomentar la conciencia, antropológica, ecológica, cívica, terrenal y espiritual del individuo; y también a enseñar la comprensión entre las personas como condición y garantía de la solidaridad intelectual y moral de la humanidad.

En este contexto de variadas transformaciones es menester que los individuos cuenten con mecanismos idóneos que les permitan potenciar sus capacidades creativas, así como descubrir sus limitaciones en el ámbito cognoscitivo, las cuales pueden inhibirlos de alcanzar mayor productividad y competencia en las labores que realizan; en este marco cobra importancia el uso oportuno y suficiente de datos e informaciones que lleven a los sujetos a expandir sus conocimientos, habilidades y destrezas; es decir, que les proporcionen el instrumental teórico-metodológico necesario para desempeñarse óptimamente en el contexto al que pertenecen.

\section{Algunos postulados constructivistas}

Según Flórez Ochoa $^{2}$ el constructivismo es una doctrina pedagógica que plantea como postulado fundamental que el aprendizaje humano es una construcción mental progresiva que permite lograr avances en los niveles de complejidad, diversidad e integración del sujeto, mediante la activación de sus estructuras y esquemas previos; de esta manera, sostiene, se transforman los elementos cognitivos presentes en él y se incrementa su capacidad

1 E. Morin. (2000). Los siete saberes necesarios a la educación del futuro. Caracas: UNESCO/IESALC.

2 F. Díaz-Barriga, y G. Hernández. (2002). Estrategias docentes para un aprendizaje significativo: una interpretación constructivista. ${ }^{a}$. Ed. México: McGraw-Hill. 
intelectual-pragmática. Desde sus inicios el constructivismo como corriente epistemológica se preocupa por discernir los problemas referidos a la formación del conocimiento en el ser humano.

En una concepción más globalizante, Carretero (citado por Díaz-Barriga y Hernández) $)^{3}$ señala que el constructivismo es el postulado que sostiene que el individuo mismo es una construcción propia que se va gestando paulatinamente como resultado de la interacción entre el ambiente y sus disposiciones internas, por lo que el conocimiento no es una copia fiel de la realidad, sino una construcción individual del ser humano, estructurada a partir de los esquemas que ya posee y de su relación con el contexto que lo rodea. Por ello, la finalidad de la educación que se imparte bajo este enfoque es promover los procesos de crecimiento personal del alumno en el marco de la cultura del grupo al que pertenece.

En esta dirección teórica, el constructivismo le adjudica al sujeto que aprende un rol protagónico en la construcción del conocimiento, por cuanto postula la existencia y prevalencia de procesos activados por un individuo aportante que mediante su labor, rebasa lo que le ofrece su entorno; es decir, el sujeto selecciona, organiza y transforma la información que recibe de diversas fuentes, establece relaciones entre dicha información y sus conocimientos previos y construye nuevos significados.

Flórez Ochoa, ${ }^{4}$ puntualiza sus características señalando que el constructivismo se apoya en la estructura conceptual que cada sujeto posee; prevé el cambio conceptual que se espera alcanzar en la estructura del aprendiz; confronta las ideas y conceptos afines nuevos con los existentes en la mente del hombre; y propicia la aplicación o funcionalidad de lo aprendido a situaciones concretas permitiendo con ello la transferencia.

Para esto se hace necesario generar un proceso de reflexión en el aprendiz, que motive el cuestionamiento de su saber actual y despierte deseos de expandirlo, que propicie la formulación de preguntas demandantes de nuevas respuestas e impulse su involucramiento deliberado en el proceso de enseñanza.

Bajo esta óptica constructivista, González y Novak ${ }^{5}$ señalan que el pensamiento científico, por su carácter evolutivo, se fundamenta en esquemas flexibles y abiertos al constante cuestionamiento de los saberes alcanzados, así como al reconocimiento de nuevas relaciones entre conceptos, reestructuraciones teórico-procedimentales y saltos creativos.

3 R. Flórez Ochoa (1995). Hacia una Pedagogía del Conocimiento. Colombia: McGraw-Hill.

4 Flórez Ochoa. op. cit.

5 F. González y J. Novak (1993). Aprendizaje Significativo. Técnicas y Aplicaciones. Argentina: Cincel. 
Lo expuesto se resume en lo que Driver (citado por Porlán) ${ }^{6}$ señala como los principios constructivistas del aprendizaje, que son:

- Lo que hay en el cerebro del que va a aprender tiene importancia

- Encontrar sentido supone establecer relaciones

- Quien aprende construye activamente significados

- Los estudiantes son responsables de su propio aprendizaje (p. 92)

\section{El aprendizaje del hombre: identificación de algunos factores intervinientes y condicionantes}

El carácter activo y selectivo del individuo en el proceso de aprendizaje es innegable, Flórez Ochoa indica que cada sujeto, a medida que interacciona con los estímulos y señales que provienen del medio ambiente natural, va produciendo sus propias experiencias, las cuales le permiten interpretar la realidad y a su vez contribuyen a su propio desarrollo; este doble accionar fue lo que Piaget (citado por Rosas y Sebastián $)^{7}$ denominó asimilación y acomodación, que corresponden a los polos de un proceso dialéctico constante concebido como equilibración de estructuras cognitivas.

Estos conceptos también fueron retomados por Wagensberg, ${ }^{8}$ quien argumenta que cualquier individuo que pretenda comprender el mundo debe comprender el cambio y la relación entre un todo y sus partes, debe asimilar cómo los sistemas se influencian mutuamente y la forma cómo esto genera otras transformaciones. Se plantea con ello un proceso permanente de adaptación al entorno, es decir, un reajuste de las estructuras para seguir siendo compatibles con lo que las rodea.

En este sentido el aprendizaje del hombre puede constituir o bien una acción deliberada de éste para acrecentar su conocimiento sobre aspectos determinados, o el resultado de las actividades que él realiza para sobrevivir en su interacción con el entorno. Por ende, y según explican Silva y Ávila, ${ }^{9}$ los humanos crean sus propios significados y construyen sus propias interpretaciones del mundo a partir de sus experiencias e interacciones; es decir, estructuran su realidad a partir de su cosmovisión, partiendo siempre de un conocimiento previo, acumulado, conservado y multiplicado del saber de sus

6 R. Porlán (1997). Constructivismo y Escuela. 3a. Ed. Sevilla: Díada Editora. p.92.

7 R. Rosas y C. Sebastian (2001). Piaget, Vigostky y Maturana. Constructivismo a tres voces. Buenos Aires: AIQUE.

8 J. Wagensberg (1989). Ideas sobre la complejidad del mundo. 2a edición. Barcelona: Tusquest.

9 E. Silva y F. Ávila (1998). Constructivismo. Aplicaciones en Educación. 2a. Ed. Maracaibo: Astro Data. 
antecesores y contemporáneos, mediante su capacidad de simbolización, registro, interpretación e integración de datos e informaciones.

Flórez Ochoa ${ }^{10}$ agrega que en la capacidad de aprendizaje de los hombres juega un papel condicionante su estructura cognoscitiva, es decir, la forma, patrón o arquitectura que toma su cognición, y las características y organización presentes en sus conceptos y esquemas de pensamiento, los cuales le proveen los instrumentos intelectivos para aprovechar las nuevas experiencias de aprendizaje. Esto lo confirma Gowin (citado por González y Novak), ${ }^{11}$ al señalar que los sentimientos acompañan a todo acto de pensar, crean significados de la naturaleza y experiencia humana, y le imprimen preponderancia o no a determinados contenidos en función de los intereses, motivos o metas personales, talentos que desee desarrollar, y sus expectativas acerca del éxito y del fracaso, etcétera.

\section{La importancia del contexto}

en cualquier experiencia de aprehensión cognitiva

En este aparte es necesario considerar los aportes de Vygostky, quien dejó un legado teórico trascendente en el ámbito de la psicología y la educación, y entre cuyas tesis destaca el impacto que produce en el aprendiz su contexto. Tal señalamiento ha sido profundizado por otros autores como Baquero (citado por Rosas y Sebastián), ${ }^{12}$ quien señala que lo social y los instrumentos de la cultura poseen un carácter formativo sobre los procesos psicológicos superiores del individuo. También Rosa y Montero ${ }^{13}$ puntualizan que los individuos adquieren conocimiento a partir del medio social en el que se encuentran insertos, y que allí mismo realizan su contribución cognitiva colectiva.

El hombre como ser histórico ha enfrentado y articulado, a la vez, diferentes entramados sociales que según Vygostky (citado por Rosa y Montero $)^{14}$ le han permitido desarrollar procesos mentales superiores a partir de la internalización de medios culturales que regulan su conducta. Esta regulación viene dada con el propósito de mantener un equilibrio con respecto a la convivencia social, y conforma el sistema de reglas que hacen parte de la cultura, o entramado social en el que se desempeña cada persona. Estas reglas no sólo ejercen un efecto regulador de la conducta social sino que también

10 Flórez Ochoa, op. cit.

11 F. González y J. Novak, op. cit.

12 R. Rosas y C. Sebastian, op. cit.

13 A. Rosa e I. Montero (1990). "El contexto histórico de la obre de Vygostky: un enfoque socio-histórico", en Moll, Luis (Compilador), Vygostky y la Educación. Connotaciones y explicaciones de la psicología socio-histórica en la educación. Argentina: AIQUE. pp. 75-107. 
producen un efecto particular en cada individuo: generan nuevas prácticas sociales que les permiten enfrentar situaciones novedosas y producir soluciones que vienen a constituir la ciencia, vista como un conocimiento condicionado históricamente.

Partiendo de esta óptica, Rosa y Montero ${ }^{15}$ afirman que la ciencia no explica el mundo tal cual es, sino que presenta una imagen del mundo a partir de la cosmovisión que el sistema social le impone en cada momento histórico. Del mismo modo, Michel Denis (citado por Gallego-Badillo), ${ }^{16}$ comenta que la imagen mental que poseen los individuos del contexto, no debe concebirse como un calco de la realidad, sino que ha de interpretarse como una construcción psicológica nueva, que a la vez constituye una actividad constructivista, mediante la cual el individuo obtiene análogos cognitivos de la realidad. En tal sentido la historia de la ciencia juega un papel fundamental, pues a través de ella se pueden encontrar los justificativos acerca de los modos en que se han generado los instrumentos teóricos de cada época, así como las formas de verificación empírica.

Esta acción constructiva del hombre se realiza a partir de la recepción, selección y transformación de informaciones provenientes del contexto, que luego de ser comparadas con otras recibidas con anterioridad y disponibles en su memoria, establecen categorías entre ellas y, finalmente, se construyen las representaciones de la realidad.

Por tal razón, Vygostky propone descubrir las implicaciones socio-históricas y el estado actual de conocimiento del contexto en el cual ha surgido cada tesis o enunciado teórico, ya que los procesos psicológicos superiores aplicados por los individuos tienen un origen cultural; es decir, se adquieren tomándolos de la cultura, por cuanto en ella están presentes los elementos activadores del pensamiento del hombre. Esto lo confirma Gallego-Badillo cuando explica que todos los seres humanos construyen representaciones y saberes sobre sí mismos, la sociedad y la naturaleza, a partir de la tradición sociocultural, socioeconómica y sociopolítica en la cual se desenvuelven. Esos saberes poseen sus propias reglas de producción y validación, orientan las praxis existenciales y las relaciones intersubjetivas, y expresan a su vez estructuras conceptuales, metodológicas y actitudinales que siguen intenciones de intervención, control, dominio y transformación de la realidad extrasubjetiva.

Otro importante aporte de Vygotsky en el entramado de los aspectos contextuales es el concepto de "Zona de Desarrollo Próximo" (ZDP), el cual es

15 Ibid.

16 R. Gallego-Badillo (1998). Discurso constructivista sobre las tecnologías. Una mirada epistemológica. Santa Fe de Bogotá: Cooperativa Editorial Magisterio. 
definido como la distancia entre el desarrollo real del individuo y su desarrollo potencial; por supuesto que en este segmento que separa a ambos estadios de desarrollo ocurre un proceso de transmisión de informaciones entre el individuo y su contexto sociocultural, que es mediado por el uso de signos que permiten tanto la transformación del estado cognitivo del sujeto como la modificación del propio contexto.

De lo expuesto se concluye que el conocimiento es un producto ante todo social, que tiene su génesis en ese contacto del individuo con el entorno, momento a partir del cual se dispara en el interior del sujeto una suerte de procesos en cadena que lo llevan a explorar, descubrir, reconocer, comparar, describir, clasificar, analizar y sintetizar, entre otras cosas; en esencia, lo conducen indefectiblemente a incrementar su bagaje de conocimientos.

Es por esto que para el constructivismo es esencial envolver la acción educativa con elementos contextualizadores que les permitan a los sujetos transferir sus aprendizajes a tareas auténticas no aisladas de la realidad y desarrollarlas en entornos que resulten significativos para ellos. El constructivismo concibe entonces al proceso educativo como una espiral ascendente que se inicia con el sujeto y su contexto, la incorporación de elementos cognitivos que incrementen su saber, y de nuevo se promueve que el sujeto intervenga positivamente en el contexto, utilizando para ello las herramientas teóricometodológicas adquiridas durante el proceso de enseñanza aprendizaje.

\section{Aprendizajes significativos}

Díaz-Barriga y Hernández ${ }^{17}$ definen el aprendizaje significativo como "aquel que conduce a la creación de estructuras de conocimiento mediante la relación sustantiva entre la nueva información y las ideas previas de los estudiantes". Lo cual posibilita la adquisición de grandes cuerpos de contenidos integrados, coherentes, estables y que poseen sentido para el individuo.

Entre las ventajas del aprendizaje significativo que destacan los autores citados está que la información aprendida significativamente sea retenida por más tiempo, extienda el conocimiento, y permita incluir con mayor facilidad aprendizajes posteriores a la vez que los aprendices ejerciten los procesos de integración, reformulación y reordenamiento conceptual, mediante la construcción de sus propios enlaces.

Moreira (citado por González y Novak) ${ }^{18}$ agrega que la retención de un conjunto de conceptos implica sobre todo la adquisición de un conjunto de significados, los cuales pueden ser de dos tipos: lógicos y psicológicos. Los 
primeros aluden a la naturaleza del material de aprendizaje: cuando éste puede relacionarse de manera sustantiva y no arbitraria con ideas preexistentes en la estructura cognitiva del sujeto; y los segundos se refieren a la experiencia cognoscitiva individual de cada alumno en función de su carácter y temperamento. En tal sentido, es necesario viabilizar intencional y sustancialmente la relacionalidad de ambos significados para que los materiales resulten de interés, y el aprendiz pueda transformar el significado lógico en psicológico.

Lo que se pretende con el aprendizaje significativo es vincular efectivamente los nuevos elementos conceptuales con los conocimientos que el individuo ya posee en su estructura cognitiva, y esto se logra cuando el aprendiz le atribuye significancia psicológica al material que se le presenta. Indican los autores en cuestión que este nexo que se establece, genera modificaciones tanto en el nuevo concepto como en los existentes, lo cual explica por qué al momento de recordar alguna información aprendida bajo este esquema, el individuo no maneja literalmente los datos tal cual fueron suministrados, sino que reelabora el discurso en función de los elementos presentes en su estructura cognitiva, y esto genera variaciones de forma pero no en la esencia del contenido.

Ausubel y Novak (citados por González y Novak) ${ }^{19}$ señalan que existe una jerarquización en nuestra estructura cognitiva a partir de la cual se estratifican los conceptos aprendidos; algunos de los cuales se consideran generales o inclusores y otros más específicos y/o subordinados. De aquí se desprende la clasificación de los aprendizajes que proponen estos autores, la cual considera que existen aprendizajes supraordenados, que se producen cuando se aprende un concepto más abstracto o general; y aprendizajes combinatorios, que se propician cuando los nuevos contenidos se relacionan con otros más generales existentes ya en la estructura cognitiva.

Basado en estas premisas Novak puntualiza la necesidad de clarificar los conceptos y reconocer la forma como éstos evolucionan y cambian en función de las experiencias, ambiciones, expectativas y retos de las nuevas generaciones; de esta manera el individuo que aprende personal e idiosincráticamente, puede establecer conexiones adecuadas entre temas de diferentes áreas del conocimiento, el cual es público y compartido.

En esencia, los autores citados sostienen que

el aprendizaje significativo es la base sobre la que descansa la integración constructivista del pensamiento, los sentimientos y los principales actos que conducen al enriquecimiento humano. 
Como su nombre lo indica, el enfoque socio-psicológico considera tanto los rasgos sociales como los psicológicos derivados del desempeño del individuo, con el propósito de estudiar sus necesidades de información y de aportar insumos de interés que le permitan a los diferentes tipos de unidades o servicios de información, actuar con mayor pertinencia en la identificación y satisfacción de dichas necesidades; las cuales según Calva ${ }^{20}$ constituyen un fenómeno que continuamente late en el interior del hombre contemporáneo.

Nuñez ${ }^{21}$ indica que este enfoque se basa en categorías, leyes, conceptos, métodos y medios, y se centra en el estudio de las necesidades de información de los usuarios entendiendo que este término designa, según Cañón, ${ }^{22} \mathrm{Sanz}^{23}$ e Izquierdo, ${ }^{24}$ a toda aquella persona que necesita y busca información para el desarrollo continuo de sus actividades, y para ello acude a sistemas o unidades de información, o hace uso de productos y servicios de información en los cuales pueda encontrar respuesta a sus necesidades, demandas y expectativas. En palabras de Hernández, ${ }^{25}$ el usuario es el protagonista principal de la trama informativa, por cuanto es él quien solicita, analiza, evalúa y transforma la información en conocimiento.

Responder a tales requerimientos exige, según Cañon, ${ }^{26}$ que los profesionales al frente de los organismos encargados de suministrar la información, conozcan y analicen, cualitativa y cuantitativamente, los factores que intervienen en la relación usuario-información, de modo que los procesos, recursos, esfuerzos y potencialidades invertidos en la planeación, montaje y oferta de los productos y servicios, tengan "una razón de ser". Esto se consolida con mayor fuerza en la actualidad, por cuanto las tendencias administrativas en las "organizaciones inteligentes" se orientan hacia el incremento de la calidad

20 J. Calva (1996). "Las necesidades de información de las comunidades científicas dentro del Programa de Formación de Recursos Humanos en Servicios Bibliotecarios y de Información para la integración de la red de Bibliotecas del Subsistema de Humanidades y Ciencias Sociales", (notas) en Investigación Bibliotecológica: Archivonomía, Bibliotecología e Información, 10 (20), enero-junio, 33-36.

21 I. Nuñez (1991-a). Perspectivas de acción, educación y entrenamiento para los trabajadores de la información. Bases. Socio-psicológicas. Papel de trabajo. Cuba

22 N. Cañón, (s.f). El usuario de la información. Segunda parte. Recuperado el 4 de diciembre del 2002 en http://www.Lasalle.edu.co/ audiovis/lib_sociales/sociología-de-la-información.htm.

23 E. Sanz (1994). Manual de estudios de usuarios. Madrid, España: Pirámide.

24 M. Izquierdo (1999). "Una aproximación interdisciplinar al estudio del usuario de información: bases conceptuales y metodológicas”, en Investigación Bibliotecológica: Archivonomía, Bibliotecología e Información, 13 (26), enero-junio, 112-134.

25 P. Hernández (1993). "El perfil del usuario de información", en Investigación Bibliotecológica: Archivonomía, Bibliotecología e Información, 7 (15), julio-diciembre, 16-22. 
de los servicios y productos que se ofrecen, para lo cual se toman como base las características, comportamientos, actitudes particulares, necesidades y expectativas de los mercados objetivos. De esta manera pues, las unidades y servicios de información, cualquiera sea su tipo, deben ofrecer servicios de calidad basados en rasgos específicos de sus usuarios, por cuanto éstos constituyen, según Izquierdo, ${ }^{27}$ el eje central del sistema de información.

De acuerdo con Hernández, ${ }^{28}$ Calva, ${ }^{29}$ e Izquierdo, ${ }^{30}$ en el delineamiento de estos rasgos intervienen factores de índole individual y contextual; los primeros se refieren a la dimensión cognitiva, emocional y/o afectiva del sujeto; es decir, lo interno: experiencias acumuladas, actitudes, valores, habilidades en el análisis de la información, etcétera. Los factores contextuales enfatizan lo social y lo sociodemográfico, y por ende lo externo al individuo, a saber: la categoría profesional que ocupa, la situación social y política, la rama de actividad a la que se dedica, las condiciones organizativas típicas y circunstanciales en las que ejecuta sus actividades, etcétera. Lo esencial de esto es que se pone en evidencia la importancia del contexto en la configuración de las necesidades, y ello puede ser entendido a partir de la explicación hecha por Vygotsky, precursor del constructivismo, quien postulaba que el entorno inculca en el individuo una estructura cultural que permea su cosmovisión y por ende lo lleva a delinear necesidades de diversa índole.

Por consiguiente el conocimiento de las necesidades de información debe partir irremisiblemente de un diagnóstico que no debe pretender alcanzar un nivel de exhaustividad total, pero sí hacer posible un acercamiento más certero al mundo subjetivo propio de cada ser humano, lo cual se logra construyendo perfiles de usuarios, definidos por Hernánde ${ }^{31}$ como el conjunto de rasgos distintivos que caracterizan al usuario y sirven de base a las unidades y servicios de información para planear las acciones a seguir. Izquier$\mathrm{do}^{32}$ señala que la importancia de dichos perfiles es tan elevada que no puede haber una aproximación al usuario sin establecer un perfil definido, el cual en ningún momento busca ser definitivo puesto que las necesidades de información de cualquier sujeto son fluctuantes y complejas, y además cambian en función de las tareas que realiza tal sujeto, y del objetivo y alcance de las tareas.

27 M. Izquierdo, op. cit.

28 P. Hernández, op. cit.

29 J. Calva (1995). "Surgimiento y manifestación de las necesidades de información de los investigadores”, en Investigación Bibliotecológica: Archivonomía, Bibliotecología e Información, 9 (19), julio-diciembre, 17-29.

30 M. Izquierdo, op. cit.

31 P. Hernández, op. cit

32 M. Izquierdo, op. cit 
Como complemento de los perfiles de usuarios, Núñez, ${ }^{33}$ indica que además se deben conformar categorías de usuarios en las que se jerarquicen los grupos identificados en función de variables como la trascendencia de las decisiones, el nivel del potencial creador, la función que desempeña el usuario como transmisor de la información y las perspectivas de desarrollo futuro; es decir, identificar también la trascendencia social presente y futura de las acciones de los individuos.

En este escenario, el enfoque socio-psicológico propuesto por varios teóricos en materia de usuarios, entre ellos Núñez, ${ }^{34}$ Hernández, ${ }^{35}$ e Izquierdo, ${ }^{36}$ se fundamenta en dos premisas básicas. Primera: toda necesidad de conocimiento que presenta un individuo obedece primordialmente a su configuración psicológica y a las demandas que le impone su contexto de acción inmediato, porque de éste deviene su cúmulo de experiencias y gran parte de sus conocimientos previos, los cuales incesantemente ameritan ser enriquecidos para continuar él siendo competente. Segunda: las unidades y servicios de información, llamadas por Pirela ${ }^{37}$ "organizaciones de conocimiento", desarrollan una función pedagógica esencial en la sociedad, por cuanto su acción responde a la satisfacción de ciertas necesidades intelectivas del individuo, lo cual repercute en el desarrollo de su potencial cognitivo, y desarrolla en éste un proceso de crecimiento sostenido que ha de repercutir positivamente en su desempeño social.

\section{Orientación de las necesidades de conocimiento de los usuarios y el rol de las unidades de información}

Para Nuñez et.al., ${ }^{38}$ Izquierdo $^{39}$ y Hernández ${ }^{40}$ las necesidades de conocimiento de cualquier individuo han de ser interpretadas, en primera instancia,

33 I. Nuñez. (1990-a, septiembre). Determinación y caracterización de las categorías o grupos de usuarios para el proceso educativo-informativo. Ponencia presentada en el $45^{\circ}$ Congreso y conferencia de la Federación Internacional de la Información y Documentación FID. La Habana.

34 I. Nuñez (1990-c). "Bases teóricas y rasgos fundamentales del enfoque socio-psicológico del servicio informativo-bibliotecario", en Nuñez, Israel (Compilador). INFORMACIÓN. Aspectos socio-psicológicos. Publicaciones IDICT (vol. I). La Habana. (Cuba) pp. 11-40.

35 P. Hernández, op. cit. / Hernández, P. (2001). "La producción del conocimiento científico como base para determinar perfiles de usuarios", en Investigación Bibliotecológica: Archivonomía, Bibliotecología e Información, 15 (30), enero-junio, 29-64.

36 M. Izquierdo, op. cit.

37 J. Pirela (2004). Los procesos de mediación en organizaciones de conocimiento de la cibersociedad. Tesis doctoral sin publicación, Universidad del Zulia, Venezuela.

38 I. Núñez; E. González; Y. Braojos y M. Sierra (1990). "La función pedagógica del sIB a través del trabajo con los grupos dinámicos de ICT”, en Núñez, Israel (Compilador), INFORMACIÓN. Aspectos socio-psicológicos. Publicaciones IDICT (vol. I). La Habana. (Cuba) pp.5-26.

39 M. Izquierdo, op. cit. 
en función de su actividad científico-productiva, porque es allí donde reside principalmente la razón que lo motiva a obtener y asimilar conocimientos; de este modo se establece una interrelación entre el Hacer y el Conocer. El primero delínea los intereses, valoraciones, posiciones del sujeto; y el segundo es a la vez origen y fin de aquél, por cuanto el perfil profesional y/o laboral está determinado por la formación recibida, por el aprendizaje obtenido, por el desarrollo de competencias que capacitan al interesado para desempeñarse como ente productivo que ejerce ciertas prácticas y roles; y a la vez, el Conocer continúa perfeccionando el Hacer mediante la asimilación de nuevos conocimientos y la reintroducción de éstos en las labores del progreso científico-tecnológico. Esto lo confirma Izquierdo ${ }^{41}$ al indicar que el usuario prefiere la información que se asocia con su formación, posición jerárquica, relaciones laborales, condiciones materiales, hábitos de trabajo, condicionantes cognitivas, situacionales, etcétera.

En función de todo esto los roles que deben desempeñar las unidades de información han experimentado cambios acelerados, originados por las exigencias de conocimiento de las comunidades de usuarios, para quienes ya no es suficiente el suministro de datos e informaciones, sino que demandan la agregación de valor a las fuentes documentales; es decir, la transformación de estos recursos en conocimiento; tal como lo señala Rodríguez (citada por Nuñez), ${ }^{42}$ quien indica que el rol de las unidades de información se ha desplazado del proveedor/transformador; hacia el generador secundario de conocimiento. Otro factor de alta incidencia es el uso intensivo de las tecnologías de información y comunicación (TIC), las cuales han trastocado la esencia misma del documento, así como los procesos de análisis que otrora se aplicaban para facilitar el acceso a su contenido.

En estos momentos asistimos a la transformación de la imagen y las funciones de las unidades y servicios de información, porque su énfasis debe apuntar hacia el fortalecimiento de las estructuras cognitivas del individuo, considerado ya no como el último eslabón de la cadena documental, sino como un ente activo y activador de nuevas prácticas en la dinámica que se establece entre el usuario y los entes encargados de proveer conocimientos.

Por consiguiente el conocimiento que los servicios de información posean acerca de las necesidades de sus usuarios no debe ser intuitivo, empírico, hipotético o derivado del estudio de demandas intermitentes, ya que esto traería como consecuencia un aislamiento de las necesidades reales y crearía además una barrera comunicativa que podría resultar infranqueable. Es preciso entonces desplegar estudios de usuarios, cuyo propósito, según

41 M. Izquierdo, op. cit.

42 I. Nuñez (1991-a), op. cit. 
Hernández, ${ }^{43}$ Calva ${ }^{44}$ e Izquierdo ${ }^{45}$ sea obtener un mayor conocimiento sobre el proceso de comunicación que se establece entre los usuarios y las unidades y servicios de información, así como analizar cualitativa y cuantitativamente la interacción entre éstos y las fuentes de información disponibles, a la vez que los aspectos cognitivo y contextual propios del sujeto; es decir, sus rasgos socio-psicológicos, los cuales delínean su modelo de mundo, y por ende inciden en sus preferencias informativas y formativas. Todo ello habrá de hacerse mediante el empleo simultáneo de diferentes técnicas de investigación social, y la combinación de varios instrumentos, ya que según Calva, ${ }^{46}$ Martín y Sanz, ${ }^{47}$ esta práctica plural ofrece un acercamiento más objetivo y además permite validar los resultados obtenidos por cada método utilizado, con el fin último de concebir formas, procesos y servicios ajustados a los requerimientos reales.

El énfasis en la dimensión cognitiva del sujeto es parte de los cambios que se están gestando al pasar de la sociedad de la información a la sociedad del conocimiento, por cuanto en esta última existe una prevalencia del sujeto, según indica Pineda ${ }^{48}$ que incrementa el interés por los procesos de aprendizaje y cognición, por encima de las tecnologías. Esto explica entonces por qué en los estudios de usuarios ha tomado auge el interés por entender la forma como el hombre asimila, comprende e interpreta la información, los procesos de razonamiento, el análisis y la síntesis que aplica para aprehenderla. Todo lo cual permite diseñar estrategias para representar y transmitir la información de un modo más eficaz.

Ante las demandas de este escenario, Sanz ${ }^{49}$ indica que el empleo de técnicas de muestreo, mediante las cuales los resultados y conclusiones obtenidos a partir del estudio de necesidades de información de usuarios reales se extrapolan para toda la población, genera sesgos que empañan las justas proporciones y particularidades de las necesidades existentes. Por otro lado, Nuñez ${ }^{50}$ explica que han venido gestándose cambios orientados a sustituir los servicios de información concebidos para grandes masas de usuarios —en los que prevalece el anonimato y se intuyen las necesidades- por formas de

43 P. Hernández (2001), op. cit.

44 J. Calva (1996). op. cit.

45 M. Izquierdo, op. cit.

46 J. Calva (1996), op. cit.

47 J. Martín y E. Sanz (2001). "Aplicación de dos técnicas de recogida de datos para la realización de estudios de usuarios", en Investigación Bibliotecológica: Archivonomía, Bibliotecología e Información, 15 (30), enero-junio, 194-213.

48 M. Pineda (2003). "La sociedad de la información como una sociedad en transición: Caracterización, tendencias y paradojas", en Revista de Ciencias Sociales, 9 (2), mayo-agosto. 
servicio basadas en el acercamiento progresivo a los requerimientos puntuales de información y formación.

Núñez, ${ }^{51}$ Sanz $^{52}$ y Calva ${ }^{53}$ plantean además que en virtud de los factores que pueden incidir en el delineamiento de las necesidades de información (circunstancias, estado psicológico y cognitivo del individuo, etcétera), éstas son propensas a sufrir constantes modificaciones, por lo que resulta conveniente instaurar el estudio de dichas demandas como un proceso de monitoreo permanente y periódico que ofrezca datos confiables sobre la forma en que éstos se configuran y reconfiguran; de manera que se realicen los ajustes correspondientes en los esquemas de servicio. Nuñez ${ }^{54}$ recomienda también que las unidades de información definan, dentro de su estructura de funcionamiento, un ente orgánico responsable de planificar y desarrollar diferentes estrategias que hagan posible un acercamiento sostenido a los requerimientos informativos y formativos de la comunidad de usuarios a la que se le presta servicio.

\section{Acciones de las unidades y servicios de información en la formación del individuo}

En un plano ideal, las unidades y servicios de información deben procurar incidir activamente en la formación de conocimientos, habilidades, hábitos, capacidades y rasgos positivos de la personalidad de sus usuarios, y desarrollar una actividad conjunta sistema-usuario en torno a un proceso educativoinformativo que coadyuve a alcanzar objetivos concretos en la conducta de estos usuarios de acuerdo con las deficiencias o insuficiencias de formación o información que cada uno presente. Tal como lo apuntó Sander ${ }^{55}$ cuando indicó que la función última de las bibliotecas de todos los tiempos "es la actualización dinámica formativa del ser humano...". Y también Núñez, ${ }^{56}$ al señalar que todos los tipos de unidades de información ejercen de algún modo una labor educativa, en tanto que facilitan el acceso al conocimiento que

51 I. Nuñez (1992). "Guía metodológica para el estudio de las necesidades de formación y de información de los usuarios o lectores", en Revista Ciencias de la Información, (23), 2, pp. 118-129.

52 E. Sanz, op. cit.

53 J. Calva (1996), op. cit./ Calva, J. (2002). "La investigación sobre las necesidades de información", (comentario) en Investigación Bibliotecológica: Archivonomía, Bibliotecología e Información, 16 (33), julio-diciembre, 3-4.

54 I. Nuñez (1991-b). "Metodología para la introducción del enfoque socio-psicológico de las entidades de información!, en Revista Ciencias de la Información, 4, pp. 10-20.

55 S. Sander (1991). "La función docente de la biblioteca y del bibliotecario en el contexto académico universitario: Una cuestión de principios”, en Revista Interamericana de Bibliotecología, 14 (2), 25-34. p.26.

56 I. Nuñez (1992), op. cit. 
los individuos requieren y ello repercute indefectiblemente en su formación intelectual.

El término "formación", desde la perspectiva de los estudios de usuarios ha sido definido por Verdugo ${ }^{57}$ como el conjunto de actividades pedagógicas orientadas al óptimo aprovechamiento de los recursos de información que el usuario necesita para solucionar algún problema o satisfacer alguna demanda académica o de otra índole. En función de esta definición se entiende que la labor de formación de usuarios que desarrollan las unidades y servicios de información supone configurar en el individuo las competencias cognitivas, instrumentales y afectivas necesarias para acceder a las fuentes documentales más idóneas en virtud de su necesidad de información, quiere decir esto que en la medida que un individuo esté más formado en estos aspectos, será más capaz de identificar y ubicar los recursos informativos de su interés. Se establece así una relación proporcional entre el nivel de formación recibido por el usuario, y el nivel de dependencia que éste pueda tener hacia la labor de mediación realizada por el especialista de información.

Apunta Verdugo, ${ }^{58}$ que al igual que todo proceso de formación parte de los conocimientos previos que posee el individuo, en el caso de las unidades y servicios de información, también es preciso considerar el nivel educativo del sujeto con el propósito de generar y aplicar las estrategias específicas adaptadas al desarrollo cognitivo alcanzado. Lo esencial en este aspecto es propiciar el acercamiento deliberado del individuo a formas sofisticadas de acceso a la información, en lo cual convergen dos dimensiones: por un lado, es preciso que el propio usuario sepa identificar con precisión sus verdaderos requerimientos de información, y esto tiene que ver con la concientización acerca de las propias necesidades; es decir, que el proceso de formación desplegado por las llamadas "organizaciones de conocimiento" debe permitirle al sujeto autodescubrir sus carencias informativas y formativas, o al menos exteriorizarlas con claridad; por el otro, es necesario instruir a los usuarios sobre las estrategias de búsqueda, utilizando las TIC, y hacer de su conocimiento el universo de fuentes físicas y virtuales que tiene a su disposición en su área de interés. Estos aspectos coinciden con los señalados por Gietz (citado por Verdugo), ${ }^{59}$ en cuanto a la sensibilización del usuario para que conozca sus necesidades de información, y la dotación de las herramientas que le facilitan al acceso a ésta.

57 J. Verdugo (1993). "Hacia un concepto de formación de usuarios y propuesta de un programa”, en Investigación Bibliotecológica: Archivonomía, Bibliotecología e Información, 7 (15), julio-diciembre, 4-15.

58 J. Verdugo, op. cit.

6259 J. Verdugo, op. cit. 
Con respecto a la dimensión actitudinal del individuo, Nuñez $z^{60}$ agrega que las unidades y servicios de información deben forjar una actitud de búsqueda y utilización constante de la información como medio para el autoaprendizaje, la solución de problemas y su aportación al desarrollo. Se debe enfatizar el despliegue de competencias instrumentales que le permitan al usuario descubrir, localizar, extraer, sintetizar y organizar racionalmente la información, tomando como base sus necesidades; es decir, aquellas que contribuyan a la formación integral de las cualidades, concepciones, actitudes y convicciones que deben caracterizar a un científico, especialista o ciudadano común.

En palabras de Verdugo, ${ }^{61}$ la calidad de la formación impartida a los usuarios se traduce en una autosuficiencia informativa de éstos, lo cual redundará en el incremento de sus oportunidades de integración social manifestada en mayor participación, facilidad en la adaptación y el desarrollo general de sus posibilidades de éxito.

\section{CONSTRUCTIVISMO Y ENFOQUE SOCIO-PSICOLÓGICO: PÁMPANOS DE UNA MISMA VID}

La complejidad innata del ser humano lo lleva a incursionar en formas distintas de aprehender los elementos de su entorno, a reelaborarlos a partir de sus interpretaciones y a generar constructos nunca acabados que pretenden mitigar temporalmente los incesantes cuestionamientos que se plantea en su devenir histórico. Hoy se ha abierto con mayor fuerza el espacio a la duda y a la incertidumbre, se ha hecho manifiesta la incompletud de los saberes, y ello ha despertado en el hombre el deseo de conocer, de profundizar en aquello que hasta ahora creía haber conocido, pero que a la vez lo amenazaba con serle desconocido, en virtud de la masiva interconexión e intercambio de conocimientos que prontamente convierten en obsoleto aun lo más novedoso.

En esta era planetaria en la que actualmente nos encontramos, el acceso a la información adquiere nuevos matices por cuanto las necesidades intelectuales son más ambiciosas y no están adscritas a una territorialidad particular sino que se contextualizan en lo global. Ante esta realidad es necesario que todos los entes creados para satisfacer necesidades de índole intelectual, tales como las instituciones educativas y las unidades y servicios de información,

60 I. Nuñez (1990-d). "La función pedagógica del sistema informativo bibliotecario como sistema de objetivos, de instituciones y de operaciones, para el proceso educativo-formativo", en Núñez, Israel (Compilador). INFORMACIÓN. Aspectos socio-psicológicos. Publicaciones IDICT (vol. I). La Habana. (Cuba) pp. 41-69.

61 J. Verdugo, op. cit 
orienten su acción hacia el desarrollo de productos y servicios cónsonos con este sentir social, y enfaticen la multidimensionalidad no sólo de los saberes sino también de las formas de acceso a ellos. Existe un agotamiento de los esquemas tradicionales y rígidos de funcionamiento en estas organizaciones, y en cambio surgen propuestas orientadas a replantear, enriquecer y reconfigurar los métodos y formas usados, desde una óptica flexible y permeable a distintas influencias y matices teóricos y pragmáticos.

En este caso particular, se indica una confluencia en los postulados teóricos del constructivismo y el enfoque socio-psicológico, cada uno de los cuales propicia desde su óptica la reconfiguración intelectiva del individuo, a partir de un proceso de formación e información construido desde y por el sujeto mismo; de modo que esa complejidad propia del hombre se erige en ambos constructos como el lienzo en el que se dibujan las acciones específicas desplegadas para atender las demandas cognoscitivas del sujeto.

Tanto el constructivismo como el enfoque socio-psicológico visualizan el entretejido existente entre los factores contextuales y el individuo, y la afectación mutua que se establece entre ellos; además puntualizan la importancia que tiene la carga subjetiva del hombre a partir de la cual se despliegan acciones orientadas a formarlo o informarlo. En ambos postulados el punto de partida y llegada es siempre el sujeto mismo, y específicamente la incidencia en su dimensión cognitiva, concebida como el elemento nuclear a partir del cual se construye la inteligencia particular y social mediante la intervención de la aptitud para organizar y aprehender el conocimiento.

Ambos enfoques teóricos procuran entonces la expansión social del sujeto a partir del enriquecimiento de su capacidad intelectiva y promueven el uso de las formas más idóneas de obtener y utilizar el conocimiento, considerando su validez y significancia en virtud de su pertinencia o congruencia con el contexto del individuo. En esencia se persigue que el sujeto desarrolle un aprendizaje sostenido y deliberado, en ocasiones mediado por el docente y otras por sí mismo, a través del uso de recursos de información, pero siempre asumiendo como punto de partida el microcosmos de éste, por cuanto la construcción de nuevos conocimientos comienza con la observación de acontecimientos o de objetos a través de los conceptos que ya se poseen, según señalan Novak y Gowin. ${ }^{62}$

Otro elemento integrador que se plantea en ambos enfoques es el énfasis en las formas de aprendizaje y construcción del conocimiento, en los procesos de pensamiento que el individuo aplica para agregarle valor a la información que percibe por sus sentidos y en los tipos de razonamiento que ejecuta

62 J. Novak y D. Gowin (1988). Aprendiendo a Aprender. Barcelona: Martínez Roca. Universidad de La Habana. Cuba. 
(deductivo, inductivo, abductivo) para obtener sus explicaciones. Es decir, que tanto el constructivismo como el enfoque socio-psicológico se adentran en la dimensión "meta" (prefijo griego que significa más allá de), al resaltar la importancia del metaconocimiento (relativo a la naturaleza misma del conocimiento y del conocer), del meta-aprendizaje (el aprendizaje relativo a la naturaleza del aprendizaje) y de la metaciencia (estudio de cómo se produce el nuevo conocimiento en las ciencias). El propósito de esto es optimizar el aprendizaje del individuo e incrementar sus posibilidades de crecimiento e inventiva, ayudándolo a hacerse consciente de su capacidad para controlar su propia experiencia y para regular sus relaciones con la sociedad, con la naturaleza y consigo mismo.

En general, ambos enfoques teóricos se sustentan en una función educativa en el sentido de que se proponen influir en el desarrollo armónico de los individuos, considerando su dimensión ética, estética, cultural, social, científica y psicológica. De esta forma, buscan contribuir de manera significativa a esa formación integral, compleja y holística que han de poseer los individuos que pretendan desenvolverse competitivamente en el actual contexto globalizado y cambiante. Lo cual lograrían mediante el despertar de su interés hacia la información que en principio resulta necesaria para su labor creativa, pero buscando que a la vez esté acorde con las tendencias de desarrollo social, político, económico, y cultural de su contexto local y global.

Entre el constructivismo y el enfoque socio-psicológico se configura una complementariedad innata, en tanto que los procesos de construcción del conocimiento que promueve el constructivismo son irrefutablemente potenciados mediante el uso de fuentes de información idóneas seleccionadas a partir del perfil social, psicológico, académico, laboral, y actitudinal del usuario o sujeto que aprende.

\section{El especialista de la información y el docente: protagonistas en la construcción del conocimiento}

Tanto los especialistas de la información como los docentes se involucran con la formación del individuo, ya que en ambos casos su labor se orienta a servir de facilitador u orientador de sujetos que poseen necesidades cognitivas, llámense usuarios o alumnos. Con respecto a esta dimensión formativa, Nuñez ${ }^{63}$ recomienda enfatizar la obtención y el afianzamiento de habilidades y hábitos en el uso del conocimiento, propiciar la formación de principios éticos profesionales y de convivencia, impulsar la conformación de una conciencia estética, 
y consolidar la convicción de la necesidad de elevar permanentemente la propia preparación. Todo ello demanda el uso de estrategias para comprender e interpretar correctamente las necesidades detectadas.

Por otra parte tanto el especialista de la información como el docente han de fomentar la creatividad e inventiva en el uso de la información para solucionar problemas o enriquecer el intelecto, y lograr así aprendizajes significativos. Estas propuestas pueden llevarse a cabo cuando existe en tales profesionales una actitud positiva para deslastrar las prácticas deterministas, monitorear permanentemente las diferencias individuales de los alumnos o usuarios, reflexionar sobre su propia praxis, corregir errores, abrirse a todas las corrientes del pensamiento, formarse y crecer como investigador, y sumergirse en su propia zona de desarrollo próximo y la de sus alumnos o usuarios; en esencia, habrá de mediar en la construcción de nuevos aprendizajes (Silva y Ávila). ${ }^{64}$

El propósito fundamental de la labor que desarrollan tanto los docentes como los especialistas de la información es llevar a los individuos a alcanzar un estadio intelectual superior, y con ello incrementar el capital intelectual de la sociedad, el cual se erige como un activo de creciente valor, inacabado por naturaleza y que demanda un suministro sostenido de nuevas informaciones para incrementar su crecimiento y traducirse en la generación de soluciones creativas y tomas de decisiones con mayores niveles de acierto. Esto repercute por ende en una mayor y mejor productividad en todos los sectores de la vida nacional.

\section{Conclusiones}

- El acelerado desarrollo científico y tecnológico de nuestros tiempos conspira contra la pertinencia social de la educación que le es impartida al hombre de hoy, y los constructos teóricos que se les enseñan a los estudiantes poseen una vigencia científica que se hace cada vez más corta, lo cual hace necesario diseñar e implantar estrategias que desarrollen en los individuos las habilidades cognitivas necesarias para avanzar a la par de esta carrera contra reloj. Ante este escenario el constructivismo se erige como un enfoque educativo que pretende rescatar al sujeto -protagonista central del proceso educativo- del rol pasivo al que había sido relegado bajo el esquema conductista; el énfasis de este nuevo enfoque puntualiza que todo proceso de aprendizaje cobra 
sentido y se hace significativo en la medida que se arraiga en la dimensión particular y social en la cual se desenvuelven los individuos; de este modo el constructivismo invita al sujeto a aprender a partir de sí mismo y de lo que posee, valorando sus construcciones y experiencias previas como el punto de partida para alcanzar niveles superiores de conocimiento que en principio respondan a su cosmovisión, pero que de forma progresiva propicien su crecimiento académico y personal.

- La educación basada en la perspectiva constructivista posee un anclaje profundo, en el sentido que lleva al sujeto a interiorizar los aprendizajes obtenidos mediante la contextualización de éstos en los escenarios en los que él interviene directamente; por tal razón, la calidad de esta formación es alta, ya que le provee al individuo las herramientas metodológicas y hermenéuticas para desarrollar efectivamente su labor social, y a la vez interpretar las circunstancias sobre una base firme de convicciones y valores que impiden su enajenación.

- El enfoque socio-psicológico del estudio de usuarios de las unidades de información, constituye un encuentro de teorías antropocéntricas a partir de las cuales se pretende incursionar en la identificación de las necesidades informativas del hombre, considerando el contexto de acción social en el que éste se desenvuelve, y a la vez los rasgos de su personalidad que inciden en la configuración de dichas necesidades. Este enfoque se basa en una perspectiva plural que toma en cuenta los aportes realizados por otras ciencias sociales entre las que resalta la psicología y la sociología, las cuales estudian respectivamente esa dimensión individual y contextual del hombre.

- El enfoque socio-psicológico se fundamenta en el hacer del individuo; es decir, aquello que constituye su labor o aporte social, ya que esto permea sus inclinaciones y preferencias y lo integra a grupos, lo asocia a pares, y configura en él o ella una suerte de inclinaciones y/o demandas de índole intelectual que se materializan en sus necesidades cognitivas. El desempeño social del hombre dibuja sus deseos intelectuales, porque a través de éste puede exteriorizar no sólo lo que sabe, sino también sus sentimientos, habilidades y particularidades; es decir, ese todo complejo que manifiesta su personalidad.

- El constructivismo y el enfoque socio-psicológico asumen una base teórica común que son las ciencias antropocéntricas y procuran satisfacer las necesidades intelectivas del ser humano, para lo cual toman el camino de la congruencia social en el sentido que parten de su arraigo contextual por considerarlo el elemento inspirador de dichas demandas. 
- Tanto el constructivismo como el enfoque socio-psicológico asumen que la carga afectiva del individuo también aflora al momento en que éste manifiesta necesidades de índole intelectual y que por tanto debe ser considerada, en el entendido de que tratará de identificar los rasgos de la personalidad que manifiestan esta carga, y la forma como tales rasgos inciden en la configuración de dichas necesidades.

- Ambos enfoques teóricos resaltan la figura del sujeto y su cotidianidad ubicándolo en el centro de las acciones que se tejen para promover su desarrollo intelectual y por ende mejorar su accionar social. Por un lado el enfoque socio-psicológico se orienta hacia la optimización de los servicios de información que ofrecen las organizaciones de conocimiento; por el otro, el constructivismo prevé el desarrollo de un proceso de enseñanza aprendizaje contextualizado, abierto y flexible.

- El desdibujamiento de las parcelas de conocimiento debe propiciar la conformación de un conglomerado de saberes que, armoniosamente integrados, propicien entre otras cosas la profundización teórica permanente, y coadyuven así al fortalecimiento de las mejores vías para el desarrollo del hombre en sus diversas dimensiones, y además provean las estrategias para su autoaprendizaje de la forma más efectiva posible. En tal sentido al sumar sus postulados teóricos el enfoque sociopsicológico y el constructivismo conforman una plataforma teórica valiosa sobre la cual puede erigirse un proceso sostenido de formación académica, cultural, laboral y personal del hombre de este tiempo.

- La construcción de enfoques inter y transdisciplinarios como el propuesto, contribuye a dar respuestas creativas a las crecientes e inimaginables demandas de la actual sociedad, la cual requiere de individuos que promuevan cambios e intervengan competitivamente en el desarrollo y progreso de los pueblos. En el caso específico del estudio de los usuarios de información, ya Nuñez ${ }^{65}$ e Izquierdo ${ }^{66}$ habían señalado la necesidad de plantear constructos interdisciplinarios que recojan distintos aportes teóricos y metodológicos (psicología cognitiva, ciencias del lenguaje, psicología social, sociolingüística, ciencias pedagógicas, entre otras), diversos modelos explicativos, y técnicas y métodos provenientes de la investigación social, con el propósito de establecer una complementariedad de enfoques que conlleve a ubicar el estudio de usuarios en un cruce interdisciplinar y conforme así una teoría suficiente y adecuada, en función de la multidimensionalidad del hombre y, por ende, de su complejidad innata. 
- Planteamientos interdisciplinarios como el propuesto demandan, entre otras cosas, que los planes de formación de los profesionales de la información incorporen elementos teóricos referidos a los aspectos psicológicos y sociales, a partir de los cuales se pueda dotar al futuro profesional de las herramientas teóricas y metodológicas necesarias para desarrollar el estudio de necesidades cognitivas en los individuos, considerando en ello lo cambiante, plural y complejo del entorno, tal como lo señala Negrete. ${ }^{67}$

- También es preciso considerar las acciones que las unidades y servicios de información deben desarrollar; en este aspecto Durrance ${ }^{68}$ indica que estas instituciones deben ser conocedoras de las tendencias sociales que prevalecen en la comunidad en la cual se encuentran insertas para de este modo ofrecer servicios congruentes con sus necesidades; asimismo habrán de vincularse con los actores influyentes, o líderes existentes en la localidad con el fin de ganar apoyo político, y hacerse de recursos y relaciones estratégicas que les permitan expandir el alcance de su acción social.

\section{BiBLIOGRAFÍA}

Calva, J. (1995). “Surgimiento y manifestación de las necesidades de información de los investigadores", en Investigación Bibliotecológica: Archivonomía, Bibliotecología e Información, 9 (19), julio-diciembre, 17-29.

Calva, J. (1996). "Las necesidades de información de las comunidades científicas dentro del Programa de Formación de Recursos Humanos en Servicios Bibliotecarios y de Información para la integración de la red de Bibliotecas del Subsistema de Humanidades y Ciencias Sociales", (notas) en Investigación Bibliotecológica: Archivonomía, Bibliotecología e Información, 10 (20), enero-junio, 33-36.

Calva, J. (2002). "La investigación sobre las necesidades de información”, (comentario) en Investigación Bibliotecológica: Archivonomía, Bibliotecología e Información, 16 (33), julio-diciembre, 3-4.

Cañón, N. (s.f). El usuario de la información. Segunda parte. Recuperado el 4 de diciembre del 2002 en http://www.Lasalle.edu.co/ audiovis/lib_sociales/sociologíade-la-información.htm.

67 M. Negrete (1993). "El papel del bibliotecólogo en el desarrollo de colecciones en la Biblioteca Universitaria", en Investigación Bibliotecológica: Archivonomía, Bibliotecología e Información, 7 (14), enero-junio, 5-7.

68 J. Durrance (1983). Bibliotecarios como agentes del cambio. Recuperado el 20 de octubre del 2004 en http://cterwilliger.com/HTML/change.html. 
Díaz-Barriga, F. Y Hernández, G. (2002). Estrategias docentes para un aprendizaje significativo: una interpretación constructivista. $2^{a}$. Ed. México: McGraw-Hill.

Durrance, J. (1983). Bibliotecarios como agentes del cambio. Recuperado el 20 de octubre del 2004 en http://cterwilliger.com/HTML/change.html.

Flórez Ochoa, R. (1995). Hacia una Pedagogía del Conocimiento. Colombia: McGraw-Hill.

Gallego-badillo, R. (1998). Discurso constructivista sobre las tecnologías. Una mirada epistemológica. Santa Fe de Bogotá: Cooperativa Editorial Magisterio.

González, F. Y Novak, J. (1993). Aprendizaje Significativo. Técnicas y Aplicaciones. Argentina: Cincel.

Hernández, P. (1993). "El perfil del usuario de información”, en Investigación Bibliotecológica: Archivonomía, Bibliotecología e Información, 7 (15), julio-diciembre, 16-22.

Hernández, P. (2001). "La producción del conocimiento científico como base para determinar perfiles de usuarios", en Investigación Bibliotecológica: Archivonomía, Bibliotecología e Información, 15 (30), enero-junio, 29-64.

Izquierdo, M. (1999). "Una aproximación interdisciplinar al estudio del usuario de información: bases conceptuales y metodológicas", en Investigación Bibliotecológica: Archivonomía, Bibliotecología e Información, 13 (26), enero-junio, 112-134.

Martín, J. Y Sanz, E. (2001). "Aplicación de dos técnicas de recogida de datos para la realización de estudios de usuarios", en Investigación Bibliotecológica: Archivonomía, Bibliotecología e Información, 15 (30), enero-junio, 194-213.

Morin, E. (2000). Los siete saberes necesarios a la educación del futuro. Caracas: UNESCO/IESALC.

Negrete, M. (1993). "El papel del bibliotecólogo en el desarrollo de colecciones en la Biblioteca Universitaria”, en Investigación Bibliotecológica: Archivonomía, Bibliotecología e Información, 7 (14), enero-junio, 5-7.

Novak, J. y Gowin, D. (1988). Aprendiendo a Aprender. Barcelona: Martínez Roca. Universidad de La Habana. Cuba.

Nuñez, I. (1991-a). Perspectivas de acción, educación y entrenamiento para los trabajadores de la información. Bases. Socio-psicológicas. Papel de trabajo. Cuba

(1991-b). "Metodología para la introducción del enfoque socio-psicológico de las entidades de información”, en Revista Ciencias de la Información, 4, pp. 10-20.

(1992). "Guía metodológica para el estudio de las necesidades de formación y de información de los usuarios o lectores", en Revista Ciencias de la Información, (23), 2, pp. 118-129. 
(1990-a, septiembre). Determinación y caracterización de las categorías o grupos de usuarios para el proceso educativo-informativo. Ponencia presentada en el $45^{\circ}$ Congreso y conferencia de la Federación Internacional de la Información y Documentación FID. La Habana.

(1990-b). "Enfoque socio-psicológico de los niveles de existencia de las necesidades informativas y sus implicaciones para una metodología de estudio", en NUÑEZ, Israel (Compilador). INFORMACIÓN. Aspectos socio-psicológicos. Publicaciones IDICT (vol. I). La Habana. (Cuba) pp. 71-88.

(1990-c). "Bases teóricas y rasgos fundamentales del enfoque socio-psicológico del servicio informativo-bibliotecario", en NUÑEZ, Israel (Compilador). INFORMACIÓN. Aspectos socio-psicológicos. Publicaciones IDICT (vol. I). La Habana. (Cuba) pp. 1140 .

(1990-d). "La función pedagógica del sistema informativo bibliotecario como sistema de objetivos, de instituciones y de operaciones, para el proceso educativo-formativo", en NUÑEZ, Israel (Compilador). INFORMACIÓN. Aspectos socio-psicológicos. Publicaciones IDICT (vol. I). La Habana. (Cuba) pp. 41-69.

Nuñez, I., González, E., Braojos, Y. y Sierra, M. (1990). "La función pedagógica del SIB a través del trabajo con los grupos dinámicos de ICT", en NUÑEZ, Israel (Compilador). INFORMACIÓN. Aspectos socio-psicológicos. Publicaciones IDICT (vol. I). La Habana. (Cuba) pp. 5-26.

Pineda, M. (2003). "La sociedad de la información como una sociedad en transición: Caracterización, tendencias y paradojas”, en Revista de Ciencias Sociales, 9 (2), mayo-agosto.

Pirela, J. (2004). Los procesos de mediación en organizaciones de conocimiento de la cibersociedad. Tesis doctoral sin publicación, Universidad del Zulia, Venezuela.

Porlán, R. (1997). Constructivismo y Escuela. 3ª Ed. Sevilla: Díada Editora.

Rosa, A. Y Montero, I. (1990). "El contexto histórico de la obra de Vygostky: un enfoque socio-histórico", Moll, Luis (Compilador). Vygostky y la Educación. Connotaciones y explicaciones de la psicología socio-bistórica en la educación. Argentina: AIQUE. pp. 75-107.

Rosas, R. Y Sebastian, C. (2001). Piaget, Vigostky y Maturana. Constructivismo a tres voces. Buenos Aires: AIQUE

Sander, S. (1991). "La función docente de la biblioteca y del bibliotecario en el contexto académico universitario: Una cuestión de principios", en Revista Interamericana de Bibliotecología, 14 (2), 25-34.

Sanz, E. (1994). Manual de estudios de usuarios. Madrid, España: Pirámide. 
Silva, E. Y Ávila, F. (1998). Constructivismo. Aplicaciones en Educación. $2^{a}$. Ed. Maracaibo: Astro Data.

Verdugo, J. (1993). "Hacia un concepto de formación de usuarios y propuesta de un programa”, en Investigación Bibliotecológica: Archivonomía, Bibliotecología e Información, 7 (15), julio-diciembre, 4-15.

Wagensberg, J. (1989). Ideas sobre la complejidad del mundo. 2a edición. Barcelona: Tusquest.

$\infty$ 